\title{
Crystal structure of tris(acetylacetonato)iron(III), $\mathrm{C}_{15} \mathrm{H}_{21} \mathrm{O}_{6} \mathrm{Fe}$, at $20 \mathrm{~K}$
}

\author{
M.-L. Hu ${ }^{\mathrm{I}}$, Z.-M. Jin ${ }^{\text {*.III }}$, Q. Miao ${ }^{\mathrm{l}}$ and L.-P. Fang ${ }^{\mathrm{I}}$ \\ I Wenzhuu Normal College, Department of Chemistry, Wenzhou, Zhejiang 325003, China \\ II Zhejiang University of Technology, School of Pharmacy, Hangzhou, Zhejiang 310014. China
}

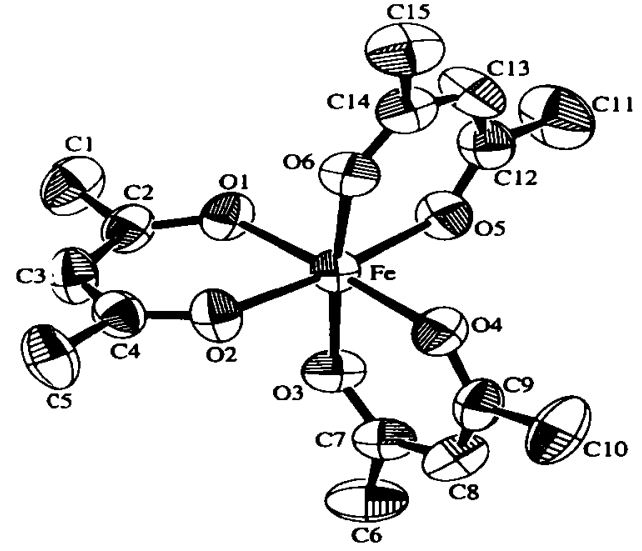

\section{Abstract}

$\mathrm{C}_{15} \mathrm{H}_{21} \mathrm{O}_{6} \mathrm{Fe},, \mathrm{Pbca}(\mathrm{No} .61), a=16.561(3) \AA$,

$b=15.434(4) \AA, c=13.578(3) \AA, V=3470.6 \AA^{3}, Z=8$,

$R_{\mathrm{gt}}(F)=0.053, w R_{\mathrm{obs}}(F)=0.059, T=20 \mathrm{~K}$.

\section{Source of material}

$3 \mathrm{mmol}$ imidazole was dissolved in $10 \mathrm{~mL}$ water, and $1 \mathrm{mmol}$ $\left(\mathrm{NH}_{4}\right)_{2} \mathrm{Fe}\left(\mathrm{SO}_{4}\right)_{2} \cdot \mathrm{H}_{2} \mathrm{O}$ was also dissolved in $10 \mathrm{~mL}$ water. The two solutions were mixed to give green precipitate, then $10 \mathrm{~mL}$ acetylacetone was added into above mixture with stirring at room temperaure. When the precipitate had entirely dissolved, the solution cianged its color from green to deep red, and the reaction mixture was filtered. The filtrate stood for six days until the red single cystals were obtained.

\section{Experinental details}

The structure was solved by Patterson method followed by Fourier synheses. $\mathrm{H}$ atoms were located in a difference Fourier map, and ther coordinates and thermal parameters were fixed during structur refinement.

\section{Discussion}

Ferric and ferrous ions exist broadly in various organisms, and play imjortant roles in bewildering array of proteins [1]. They relate closely to many functions such as $\mathrm{O}_{2}$ storage in yoglobin, $\mathrm{O}_{2}$ transport in hemoglobin, oxidation of inactivated carbonhydroge bonds in cytochrome $\mathrm{P}_{450}$, oxygen reduction in cytochromeC oxidase and so on $[2,3]$. Consequently, much interest has been focused on their complexes [4]. What is more, the $\beta$-dione find a variety of laboratory uses and many industrial applications and have contributed a lot to the present understanding of sterit pressure and conformers, prototropy and tautomerism, chelate ormation, and intramolecular bonding [5]. So we use ferric ion andacetylacetonato anion as center ion and ligand respectively

\footnotetext{
* Crrespondence author (e-mail: zhimin-j@163.net)
}

to synthesize the title complex and report its crystal structure herein.

The molecular structure of the title complex with the atom numbering scheme is illustrated in the figure (left, $50 \%$ probability displacement elipsoids). In a molecule of the title complex, six oxygen atoms from three bidentate acac anions coordinate to a ferric ion to complete the coordination geometry of a six-coordinated octahedron around the ferric ion. It is worthy to take a look at the distances and angles around ferric ion in this complex. The bond angles of $\mathrm{O}(1)-\mathrm{Fe}-\mathrm{O}(4), \mathrm{O}(3)-\mathrm{Fe}-\mathrm{O}(6)$, $\mathrm{O}(2)-\mathrm{Fe}-\mathrm{O}(5)$, are $176.4(1)^{\circ}, 175.0(1)^{\circ}, 173.5(1)^{\circ}$, respectively, which are all smaller than $180^{\circ}$. On the other hand, the bond distances of all the $\mathrm{Fe}-\mathrm{O}$, namely $\mathrm{Fe}-\mathrm{O}(1)[2.004(3) \AA], \mathrm{Fe}-\mathrm{O}(2)$ [1.996(3) $\AA], \mathrm{Fe}-\mathrm{O}(3)[1.995(3) \AA], \mathrm{Fe}-\mathrm{O}(4)$ [1.977(3) $\AA]$, $\mathrm{Fe}-\mathrm{O}(5)[1.985(3) \AA], \mathrm{Fe}-\mathrm{O}(6)[1.992(3) \AA$ ] , are not equal to each other. Therefore, the coordination geometry formed by six oxygen atoms is a distorted octahedron. $A \beta$-diketonate anion coordinate usually to a metal cation in a chelate fashion [6]. The bond distances of $\mathrm{O}(1)-\mathrm{C}(2), \mathrm{O}(2)-\mathrm{C}(4), \mathrm{C}(2)-\mathrm{C}(3)$ and $\mathrm{C}(3)-\mathrm{C}(4)$ are $1.274(6) \AA, 1.258(6) \AA, 1.381(7) \AA$ and $1.390(7) \AA$, respectively, which are in range between the single and double bond distances. It shows that there exists conjugated structure in each coordinated $\beta$-diketonate anion acac, which leads to the delocalization of electron density of the coordinated $\beta$-diketonate structure. Whereas, the annular and exocyclic $\mathrm{C}-\mathrm{C}$ distances are on average $1.385 \AA$ and $1.504 \AA$, respectively, which are the main deviations from those observed at room temperature $(1.377 \AA$ and $1.530 \AA$, respectively) [7]. The mean values of annular and exocyclic $\mathrm{O}-\mathrm{C}-\mathrm{C}$ angle for the title compound at $20 \mathrm{~K}$ are $124.2^{\circ}$ and 115.6 , respectively, and those observed previously [7] at room temperature are $125.0^{\circ}$ and 114.9 , respectively. The other bond lengths and angles are almost the same in spite of the temperature difference. All in all, three acac coordinates concurrently to a ferric ion to result a distorted octahedron of the title complex with three six-membered rings. The plane through $\mathrm{O}(1), \mathrm{O}(2)$ and $\mathrm{Fe}$ joints to the plane through $\mathrm{O}(3), \mathrm{O}(4)$ and $\mathrm{Fe}$ at an angle of $86.49^{\circ}$, to the plane through $\mathrm{O}(5), \mathrm{O}(6)$ and $\mathrm{Fe}$ at an angle of $78.91^{\circ}$. In the molecular packing, separate molecules insert with each other by the ramose six-membered ring.

Table 1. Data collection and handling.

$\begin{array}{ll}\text { Crystal: } & \text { red prismatic, size } 0.20 \times 0.20 \times 0.30 \mathrm{~mm} \\ \text { Wavelength: } & \text { Mo } K_{\alpha} \text { radiation }(0.7107 \AA) \\ \mu: & 89.13 \mathrm{~cm}^{-1} \\ \text { Diffractometer, scan mode: } & \text { Rigaku AFC7R, } \omega / 2 \theta \\ 2 \theta_{\text {max }}: & 54.98^{\circ} \\ N(h k l)_{\text {measured, }} N(h k l)_{\text {unique }}: & 3944,3744 \\ \text { Criterion for } l_{\text {obs, }} N(h k l)_{\text {gl }}: & I_{\text {obs }}>2.5 \sigma\left(I_{\text {obs }}\right), 1920 \\ N(\text { param })_{\text {refined: }} & 200 \\ \text { Programs: } & \text { SHELXS-86 [8], teXsan [9] }\end{array}$


Table 2. Atomic coordinates and displacement parameters (in $\AA^{2}$ ).

\begin{tabular}{llllll}
\hline Atom & Site & $x$ & $y$ & $z$ & $U_{\text {iso }}$ \\
\hline$H(1)$ & $8 c$ & 0.4157 & 0.7303 & 0.4703 & 0.140 \\
$H(2)$ & $8 c$ & 0.4525 & 0.7968 & 0.3974 & 0.165 \\
$H(3)$ & $8 c$ & 0.4998 & 0.7132 & 0.4238 & 0.152 \\
$H(4)$ & $8 c$ & 0.5091 & 0.6333 & 0.2803 & 0.095 \\
$H(5)$ & $8 c$ & 0.5226 & 0.5898 & 0.0974 & 0.190 \\
$H(6)$ & $8 c$ & 0.5025 & 0.5021 & 0.1475 & 0.130 \\
$H(7)$ & $8 c$ & 0.4519 & 0.5336 & 0.0582 & 0.192 \\
$H(8)$ & $8 c$ & 0.2741 & 0.4225 & 0.4359 & 0.136 \\
$H(9)$ & $8 c$ & 0.2155 & 0.3564 & 0.3878 & 0.101 \\
$H(10)$ & $8 c$ & 0.1821 & 0.4283 & 0.4565 & 0.157 \\
$H(11)$ & $8 c$ & 0.1343 & 0.3974 & 0.2450 & 0.087 \\
\hline
\end{tabular}

Table 2. Continued.

\begin{tabular}{llllll}
\hline Atom & Site & $x$ & $y$ & $z$ & $U_{\text {iNo }}$ \\
\hline$H(12)$ & $8 c$ & 0.0903 & 0.4097 & 0.0803 & 0.182 \\
$\mathrm{H}(13)$ & $8 c$ & 0.0396 & 0.4936 & 0.0913 & 0.159 \\
$\mathrm{H}(14)$ & $8 c$ & 0.1115 & 0.4909 & 0.0182 & 0.130 \\
$\mathrm{H}(15)$ & $8 c$ & 0.0528 & 0.7582 & 0.3963 & 0.151 \\
$\mathrm{H}(16)$ & $8 c$ & 0.0393 & 0.8438 & 0.3388 & 0.184 \\
$\mathrm{H}(17)$ & $8 c$ & 0.1083 & 0.8374 & 0.4157 & 0.237 \\
$\mathrm{H}(18)$ & $8 c$ & 0.1214 & 0.8773 & 0.1987 & 0.063 \\
$\mathrm{H}(19)$ & $8 c$ & 0.1707 & 0.8468 & -0.0042 & 0.103 \\
$\mathrm{H}(20)$ & $8 c$ & 0.2030 & 0.9207 & 0.0626 & 0.149 \\
$\mathrm{H}(21)$ & $8 c$ & 0.2628 & 0.8564 & 0.0137 & 0.123 \\
& & & & & \\
\hline
\end{tabular}

Table 3. Atomic coordinates and displacement parameters (in $\AA^{2}$ ).

\begin{tabular}{|c|c|c|c|c|c|c|c|c|c|c|}
\hline Atom & Site & $x$ & $y$ & $z$ & $U_{11}$ & $U_{22}$ & $U_{33}$ & $U_{12}$ & $U_{13}$ & $U_{23}$ \\
\hline $\mathrm{Fe}$ & $8 c$ & $0.25418(4)$ & $0.64276(4)$ & $0.23083(5)$ & $0.0521(4)$ & $0.0604(4)$ & $0.0429(4)$ & $0.0006(4)$ & $-0.0008(3)$ & $-0.0056(3)$ \\
\hline$O(1)$ & $8 c$ & $0.3324(2)$ & $0.7029(2)$ & $0.3203(2)$ & $0.064(2)$ & $0.075(2)$ & $0.052(2)$ & $-0.009(2)$ & $-0.004(2)$ & $-0.019(2)$ \\
\hline$O(2)$ & $8 c$ & $0.3498(2)$ & $0.5940(2)$ & $0.1605(2)$ & $0.058(2)$ & $0.092(3)$ & $0.056(2)$ & $0.011(2)$ & $-0.003(2)$ & $-0.023(2)$ \\
\hline$O(3)$ & $8 c$ & $0.2502(2)$ & $0.5443(2)$ & $0.3258(2)$ & $0.088(3)$ & $0.059(2)$ & $0.048(2)$ & $-0.003(2)$ & $-0.015(2)$ & $-0.002(2)$ \\
\hline $\mathrm{O}(4)$ & $8 c$ & $0.1786(2)$ & $0.5767(2)$ & $0.1469(2)$ & $0.067(2)$ & $0.079(2)$ & $0.052(2)$ & $-0.009(2)$ & $-0.014(2)$ & $0.001(2)$ \\
\hline$O(5)$ & $8 c$ & $0.1669(2)$ & $0.7028(2)$ & $0.3041(2)$ & $0.065(2)$ & $0.080(3)$ & $0.051(2)$ & $0.004(2)$ & $0.016(2)$ & $0.004(2)$ \\
\hline $\mathrm{O}(6)$ & $8 c$ & $0.2485(2)$ & $0.7382(2)$ & $0.1323(2)$ & $0.078(2)$ & $0.066(2)$ & $0.048(2)$ & $0.004(2)$ & $0.012(2)$ & $0.001(2)$ \\
\hline$C(1)$ & $8 c$ & $0.4476(4)$ & $0.7386(5)$ & $0.4122(4)$ & $0.100(5)$ & $0.116(5)$ & $0.085(4)$ & $-0.025(4)$ & $-0.033(4)$ & $-0.012(4)$ \\
\hline$C(2)$ & $8 c$ & $0.4083(3)$ & $0.6911(4)$ & $0.3290(4)$ & $0.062(4)$ & $0.078(4)$ & $0.055(3)$ & $-0.012(3)$ & $-0.012(3)$ & $0.002(3)$ \\
\hline$C(3)$ & $8 c$ & $0.4534(3)$ & $0.6397(4)$ & $0.2666(4)$ & $0.053(3)$ & $0.105(5)$ & $0.088(4)$ & $0.004(3)$ & $-0.016(3)$ & $-0.006(4)$ \\
\hline$C(4)$ & $8 c$ & $0.4231(3)$ & $0.5970(3)$ & $0.1844(4)$ & $0.060(3)$ & $0.075(4)$ & $0.066(4)$ & $0.008(3)$ & $0.011(3)$ & $0.002(3)$ \\
\hline$C(5)$ & $8 c$ & $0.4806(4)$ & $0.5499(5)$ & $0.1166(5)$ & $0.078(4)$ & $0.136(6)$ & $0.104(5)$ & $0.034(4)$ & $0.023(4)$ & $-0.016(5)$ \\
\hline$C(6)$ & $8 c$ & $0.2210(4)$ & $0.4147(3)$ & $0.4085(4)$ & $0.145(6)$ & $0.061(3)$ & $0.063(3)$ & $0.000(4)$ & $0.009(4)$ & $-0.001(3)$ \\
\hline$C(7)$ & $8 c$ & $0.2119(3)$ & $0.4740(4)$ & $0.3217(4)$ & $0.085(4)$ & $0.056(3)$ & $0.048(3)$ & $0.003(3)$ & $0.014(3)$ & $-0.005(3)$ \\
\hline$C(8)$ & $8 c$ & $0.1617(3)$ & $0.4512(3)$ & $0.2422(4)$ & $0.091(4)$ & $0.074(4)$ & $0.063(3)$ & $-0.025(3)$ & $0.005(3)$ & $-0.003(3)$ \\
\hline$C(9)$ & $8 c$ & $0.1483(3)$ & $0.5024(4)$ & $0.1610(4)$ & $0.054(3)$ & $0.087(4)$ & $0.056(3)$ & $-0.010(3)$ & $0.002(3)$ & $-0.012(3)$ \\
\hline$C(10)$ & $8 c$ & $0.0917(4)$ & $0.4724(5)$ & $0.0806(4)$ & $0.092(5)$ & $0.150(7)$ & $0.080(4)$ & $-0.048(4)$ & $-0.024(4)$ & $-0.016(4)$ \\
\hline$C(11)$ & $8 c$ & $0.0776(4)$ & $0.8065(5)$ & $0.3654(5)$ & $0.113(6)$ & $0.134(6)$ & $0.119(5)$ & $0.036(5)$ & $0.063(5)$ & $-0.010(5)$ \\
\hline$C(12)$ & $8 c$ & $0.1356(3)$ & $0.7759(4)$ & $0.2875(4)$ & $0.067(4)$ & $0.082(4)$ & $0.061(4)$ & $0.008(3)$ & $0.012(3)$ & $-0.012(3)$ \\
\hline$C(13)$ & $8 c$ & $0.1507(4)$ & $0.8249(4)$ & $0.2055(5)$ & $0.094(4)$ & $0.086(4)$ & $0.080(4)$ & $0.036(4)$ & $0.006(4)$ & $0.010(4)$ \\
\hline$C(14)$ & $8 c$ & $0.2047(4)$ & $0.8036(4)$ & $0.1310(4)$ & $0.082(4)$ & $0.071(4)$ & $0.046(3)$ & $-0.006(3)$ & $-0.011(3)$ & $0.001(3)$ \\
\hline$C(15)$ & $8 c$ & $0.2110(4)$ & $0.8620(4)$ & $0.0428(4)$ & $0.136(6)$ & $0.095(4)$ & $0.058(3)$ & $-0.003(4)$ & $-0.017(4)$ & $0.021(3)$ \\
\hline
\end{tabular}

\section{References}

1. Yan, S. P.; Cheng, P., Wang, Q. L.; Liao, D. Z.; Jiang, Z. H.; Wang, G. L.: Sythesis, Crystal Structure of Differous Complex and Its Reactivity with Dioxygen. Sci. Chin. B43 (2000) 405-411.

2. Bar-On, P.; Mohsen, M.; Zhang, R.; Feigin, E.; Chevion, M.; Samuni, A.: Kinetcs of Nitroxide Reation with Iron(II). J. Am. Chem. Soc. 121 (1999) 8070-8073.

3. Rose, E.; Quelquejeu, M.; Lecas-Nawrocka, A.; Vilar, A.; Ricart, G.; Collman, J. P.; Wang, Z.; Straumanis, A.: Synthesis of Porphyrins: Models of Natural Hemoprotein and Impressive Catalysts for Asymmetric Epoxidation of Olefins. Polyhedron. 19 (2000) 581-586.

4. Flavia, N.; Angela, L.; Giancarlo, M.; Carlo, P.; Vincenzo, P.; Genevieve, C.; Pierrette, B.; Daniel, M.: Hemoprotein Models Based on a Covalent Helix-Heme-Helix Sandwich. 3. Coordination Properties, Reactivity and Catalytic Application of Fe(III)- and Fe(II)-Mimochrome I. J. Biol. Inorg. Chem. 3 (1998) 671-681.
5. Voutsas, G.; Tzavellas, L. C.; Tsiamis, C.: Self-Assemblies and Supramolecular Structures in Metal-Dionates: Crystal and Molecular Structure of Bis(aquo)bis(3-cyno-2,4-pentanedionato)Zinc(II) Dihydrate, $\left[\mathrm{Zn}\left(\mathrm{H}_{2} \mathrm{O}\right)_{2}(\mathrm{NC}-\mathrm{acac})_{2}\right] \cdot 2 \mathrm{H}_{2} \mathrm{O}$. Struct. Chem. 10 (1999) 53-57.

6. Hu, M. L.; Huang, Z. Y.; Cheng, Y. Q.; Wang, S.; Lin, J. J.; Hu, Y.; Xu, D. J.; $\mathrm{Xu}, \mathrm{Y}$. Z.: Crystal Structure and Fluorescence Spectrum of The Complex [Eu(III)(TTA) 3 (phen)]. Chin. J. Chem. 17 (1999) 637- 643.

7. Iball, J.; Morgan, C. H.: A Refinement of the Crystal Structure of Ferric Acetylacetonate. Acta Crystallogr. 23 (1967) 239-244.

8. Sheidrick, G. M.: SHELXS-86. Program for the solution of crystal structures. University of Göttingen, Germany 1986.

9. teXsan: Single Crystal Structure Analysis Software. Version 1.04. Molecular Structure Corporation. The Woodlands, TX, USA 1997. 